\title{
The Affective City: Cartography of Machinic Urban Assemblages
}

\author{
Yota Passia; Panagiotis Roupas
}

NTUA School of Architecture

giotapassia@gmail.com; studioentropia@gmail.com

\begin{abstract}
While the city is generally perceived -within complexity theory and dynamic systems theory as a changing field of dense interactions that occur in a range of spatial and temporal scales, we are unable to perceive it or describe it in these terms. The main goal of our work is to redefine space - ontologically and epistemologically- in order to reveal the invisible system of its interactions. Thus, the city is represented via a connectionist model, by means of the representation of the interconnections of its various parts. The project theorizes the city as a multiplicity, a structure of spaces of possibilities while at the same time trying to establish a liaison between the city's properties, tendencies and capacities. While properties are actual and can be observed, tendencies and capacities lie in the virtual level and become actual once exercised. Their intensive cartographies map zones of varying degrees regarding the density and intensity of information that is available pointing them as the city's islands of affordances. In this framework, Hecate becomes the city's virtual map, an interactive mechanism able to decipher, map and connect the superimposed layers of significance informing the city's layout and behavior, in real time. Visualized as a network of richly interconnected nodes of varying intensities, each representing information flows between the system and the city, Hecate is introduced as a visual thinking tool to perceive them in real time.
\end{abstract}

Keywords: Connectionist Model; Urban Superscape; Affects; Intensity; System

\section{Introduction}

While space is generally perceived as a changing field of dense interactions that occur in multiple spatiotemporal scales, we are unable to perceive or describe it in these terms while at the same time we are unable to respond adequately to urban transformation patterns. The project theorizes urban space as a complex fabric of forces between relations and aims at redefining space in order to reveal the invisible system of its interactions. The above challenges are being approached through Hecate, a mechanism that is gathering significant information about the city while establishing for them a means of communication. Hecate is a virtual map and an urban infrastructural system. As a virtual map, it poses a methodology and a visual thinking tool that pushes the limits of our minds' cognitive capacities to better perceive and visualize space's intricate complexity. As an infrastructural system, it is an interactive city scape that functions as the city's nervous system. It is visualized as a network of richly interconnected nodes of varying intensities, each representing information flows between the system and the city. Hecate is itself a new map of the territory, in constant flux.

Acknowledging the limitations of our cognitive capacities to perceive the complexity of space we turn to cartography in order to extend our minds' consciousness regarding the city while at the same time expanding the city's consciousness regarding its possible futures. In this framework, the research aims at the construction of a virtual map, a mechanism capable of grasping a rich variety of possible urban configurations. Through this virtual map, we are able to both enhance our cognitive capacities via a new digital aesthetic (or aesthesis) and at the same time unlock potentialities latent in the urban environment. The virtual map both as a methodology and a visual thinking tool is a means to better understand and visualize space's intricate complexity as it unfolds at different spaces, speeds and rhythms. 
The mechanism we propose is the structure of spaces of possibilities within the city, as we seek to establish the difference between properties on the one hand and tendencies and capacities on the other. According to DeLanda (2013) their basic difference is that while the former are actual the latter have not yet been exercised and thus remain in the virtual. As they both define objective entities - here space and the city - equally and in order to gain knowledge about those entities, DeLanda (2013) proposes that one should create representations of them based on their properties, while performing interventions on them in order to force them to manifest their tendencies. In addition, one should get them to interact with other entities so that they exhibit the variety of their capacities. By superimposing the city's properties, tendencies and capacities a virtual map is produced as a means to map space in terms of both how space is now (actuality) and how space has the capacity to be (virtuality).

As the virtual map is retrieving information regarding urban space and its possibilities, we draw on specific theoretical standpoints in order to more thoroughly define them. Here, we consider the limit of urban space as the limit of its affordances or action possibilities and not the limit of its form. Gibson (1986) notes that "the affordances of the environment are what it offers the animal, what it provides or furnishes, either for good or ill. The verb to afford is found in the dictionary, but the noun affordance is not. I mean by it something that refers to both the environment and the animal in a way that no existing term does. It implies the complementarity of the animal and the environment [...]." As invariant combination of variables, affordances are meant to be objective, real and physical, unlike values which are subjective, phenomenal, and mental. As the term 'capacity' is closely related to the term 'affordance' according to Gibson (1986), it is difficult to find a better example of a concept that shifts the focus from the extensive space of properties to the intensive matrix of capacities. That said we can now move from the static extensive space to the more dynamic intensive one while grasping the variables that explain their intricate correlations.

Theorizing contemporary urban substance on a flat ontology plateau, we are allowed to pull apart its elements so that they are free to reassemble and therefore capable to produce new spatial configurations. Elements can now be related freely as long as they do not fuse together, are not engulfed by other elements or attached to an a priori constructed top-down hierarchy. As DeLanda (2013) notes "While an ontology based on the relations between general types and particular instances is hierarchical each level representing a different ontological category (organism, species, genera), an approach in terms of interacting parts and emergent wholes leads to a flat ontology, one made exclusively of unique, singular individuals, differing in spatiotemporal scale but not in ontological status". In theorizing urban space as a palimpsest of properties, tendencies and capacities, we are trying to establish the flat ontology of its spatial elements, in terms of interacting parts and emergent wholes. As dualities and their respective boundaries become obsolete, the built and unbuilt, the organic and inorganic are reevaluated in terms of their capacity to affect and be affected, while constantly entering into new urban configurations or assemblages.

We focus on spatial assemblages within urban city areas, as we believe that their latent potentialities tend to define their degree of resilience and vitality. Spatial elements such as ground, envelope, internal or external space are mapped in terms of their properties, capacities and tendencies and superimposed on the same immanent plane (Deleuze \& Guattari, 1987) where they are able to relate to one another freely. On this plane, they are allowed to interact - empathize with one another - rather than stick to their materialistic autonomy. We consider them as free radicals, ready to enter to the formation of new assemblages. Instead of mapping buildings or building typologies, we map the relations between them and their respective parts in order to enhance the multivalent properties of inhabiting within contemporary urban assemblages.-

\section{Hecate: the city's virtual map}

Drawing from Greek mythology, Hecate (/'hekəti:/; Ancient Greek: 'Ekó $\eta \eta$, Hekátē) has been the goddess that held the skeleton-key to all three possible worlds (of the living, the dead and the 
gods). She is also the means through which all traces of information - past, present and future resurface and associate anew, topologically interconnecting different levels and scales in real time. Hecate then becomes in this context the city's virtual map aiming at gathering information about the city that is significant while at the same time and most importantly establishing for them a means of communication and exchange.

The communication system proposed - draws form from Quentin Meillassoux's concept of contingency (Meillassoux, 2012) - of both machine and labor, is one that transcribes the contingent city (its tendencies and capacities) to the sensible city (its properties), thus granting us further access to the former. As Meillassoux argues there is a qualitative leap from one equilibrium state of a system to another, and therefore we cannot explain one state in terms of the physical laws of the other. In this manner, we can assume that our world is contingent, and in order to better grasp it, we need to focus not on what currently exists, but as Meillassoux puts it, on the 'perhaps'. (Meillassoux, 2012). These possible states are deriving from the current configuration, while at the same time have the ability to merge with it and reveal more properties than was there before. According to Meillassoux (2012) one could identify the latent potentialities by recognizing what is currently lacking in the current configuration.

Hecate is a registering mechanism of both actual and virtual networks of significance within the city as they develop and advance in time. Following the reconstruction of Deleuze's world and specifically his theory of sense (developed in The Logic of Sense) by Manuel Delanda, he mentions that Deleuze "clearly distinguishes "sense" from "signification" (the semantic content of words or sentences), "sense" being more connected with "significance" (the capacity of words or actions to make a difference)" (DeLanda, 2014) In this framework, the concepts of significance and difference are central in understanding the characteristics and behavior of Hecate, each intensive mapping having the capacity to differ in space and time resulting in a virtual map in flux.

Hecate maps all affordances of spatial elements within the cityscape, as they change and reorganize themselves into new assemblages. Each intensive cartography monitors one of the city's intensity zones as it is being defined by critical points of change. Hecate is an affective virtual map constantly adjusting to the city's networks as they develop into spatiotemporal data structures with the ability to store information regarding the city and use it to develop or adjust in the future. These structures are not simply formal objects or outcomes of mapping processes where data enter the virtual map; they are indicative of our computational culture and its aesthetic resonance.

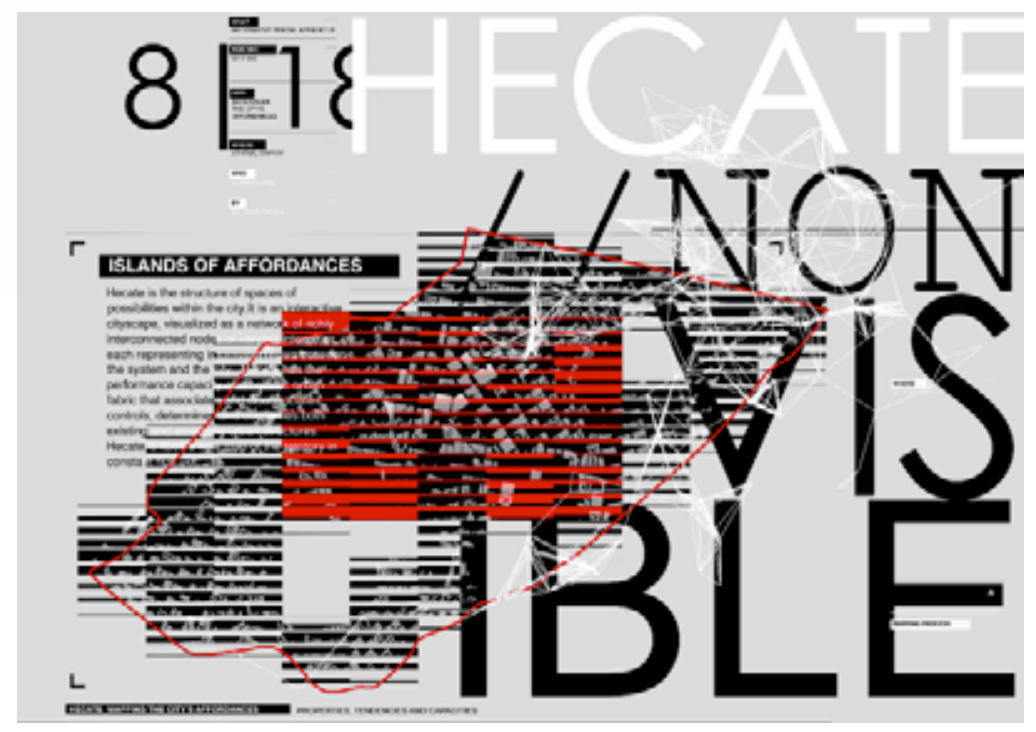

Picture 01: Virtual Map of Athens (Passia Y. \& Roupas, 2018). 
In the case study presented, a virtual map of Athens (pic.01) is constructed to reflect on the materiality of data and their variations through the representation of spatial relations amongst urban elements. Properties of architecture and the city on one hand and their tendencies and capacities on the other are equally mapped, documented and communicated anew. The topological relations of space tend to supersede the geometrical matrix of built spaces by adding more information to fixed points in space, establishing the virtual map where all points are interconnected in real time.

\section{Abstraction}

"The task of abstraction in this scenario is to liberate the virtual subject- the designated force of thought - from the trap of the material. But this liberation is conducted precisely by utilizing the resources of the material, with the aid of tendencies, properties and parameters, that determine and govern the behavior of the material system and, correspondingly, constrain the dynamic of thought, forcing it to revise its formation and to triangulate new affordances for conception and action (Reza Negarestani, 2014). Using generative algorithmic processes, we seek to construct an abstract ontogenic model that visualizes spatial correlations via a set of translating protocols where any information of the urban environment is transformed into a map of qualitative tensions. One of the major research goals is to develop an insight or knowledge regarding the complexity of underlying correlations affecting cities. A constructed landscape of formal elements - as we understand contemporary cities - is built upon perceptions that are restricting. To question preconceptions regarding the city's built reality, Hecate seeks to allow for the experience of those non-visible, non-discrete elements that define the city's identity and material reality. Via a plethora of intensive mappings within the urban context, we establish a methodology able to visualize the city's hidden properties, tendencies and capacities that have been out of sight and thus misperceived.

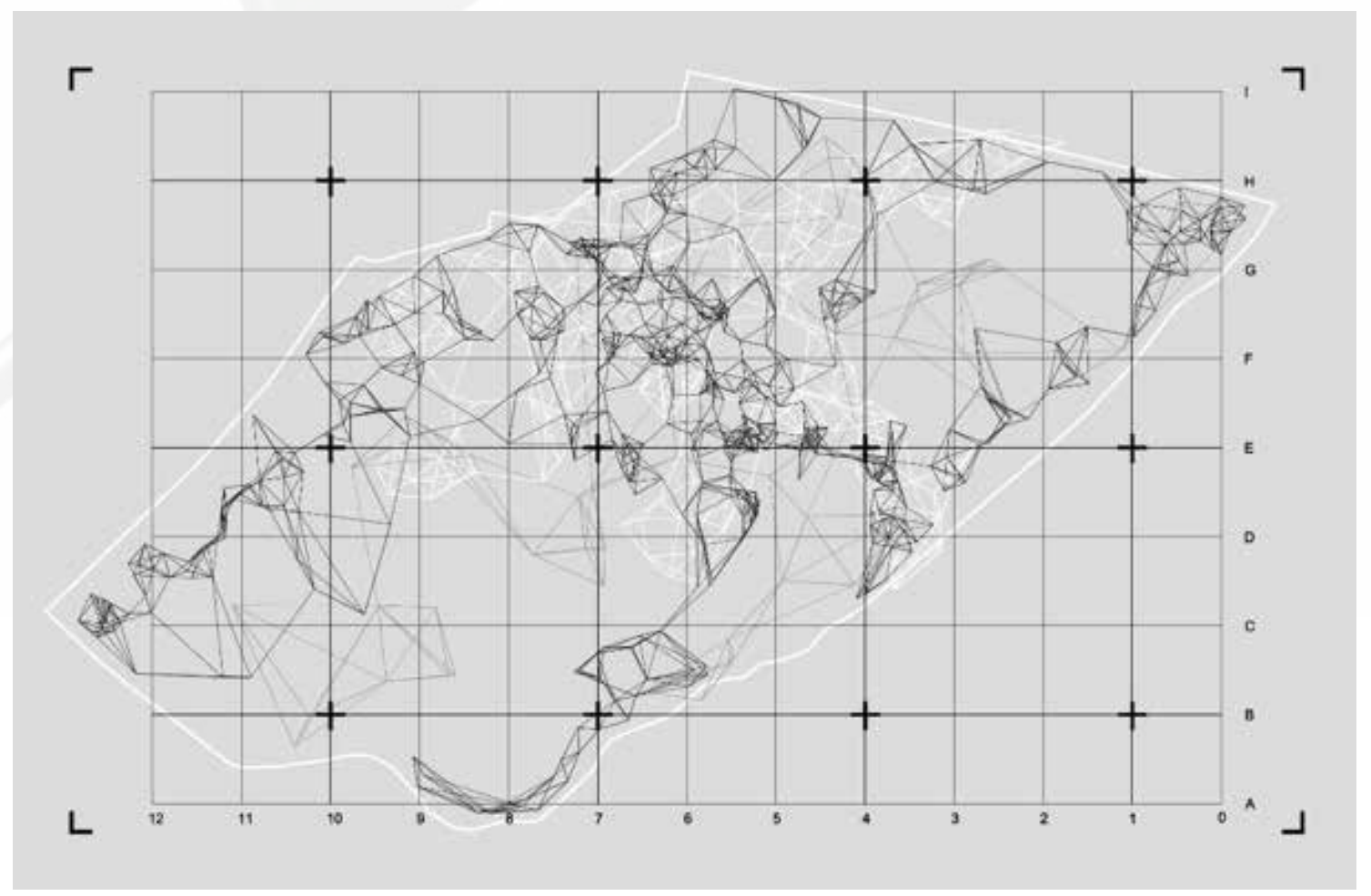

Picture 02: Information Density + Intensity maps (Passia \& Roupas, 2018). 


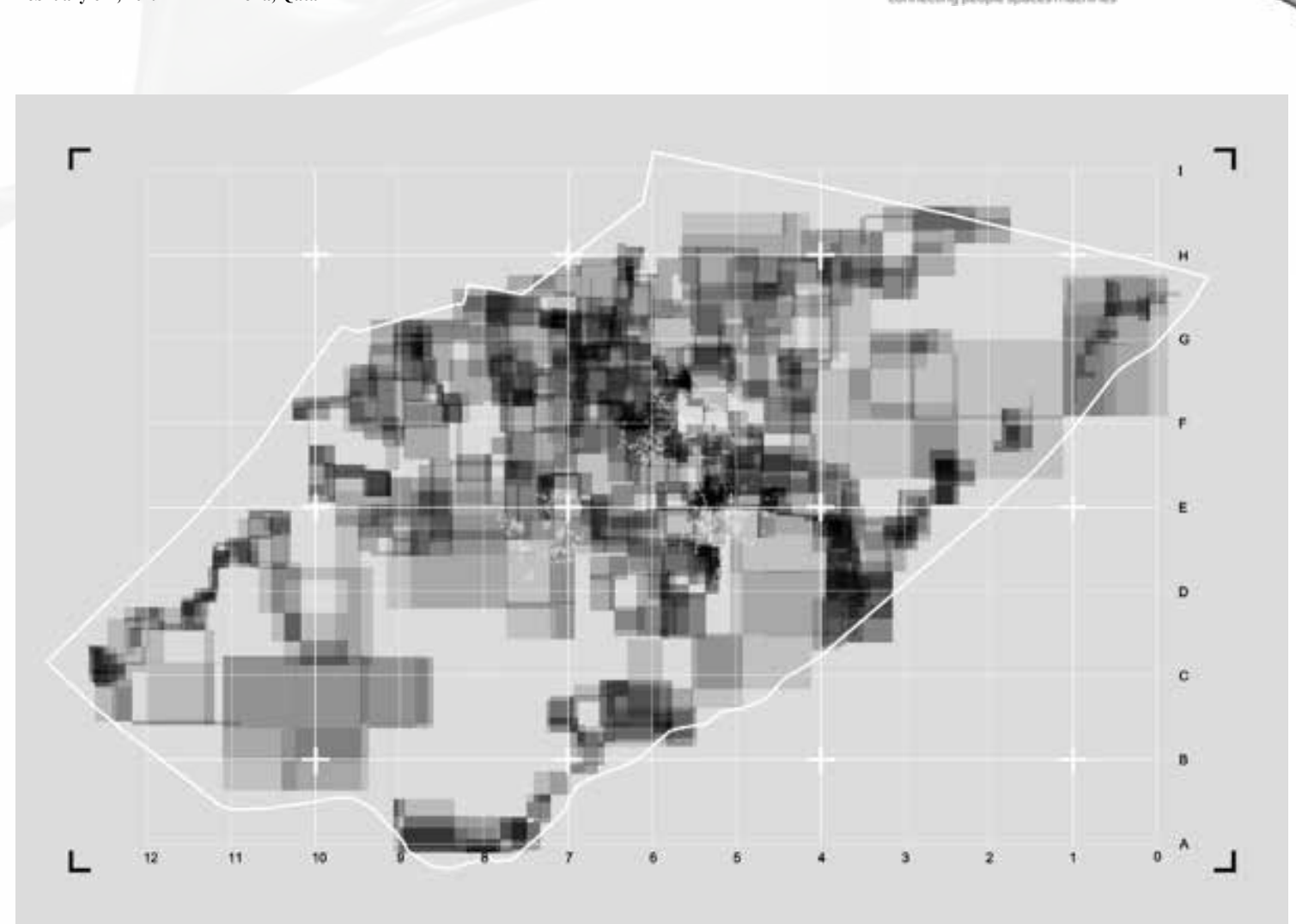

Picture 03: Islands of Affordances (Passia \& Roupas, 2018).

The complexity of information within the cityscape hinders our acquisition of cognition and knowledge. As information mapped is both highly differentiated and fragmented but also occurring at different levels, scales and speeds, Hecate actively reorganizes it by applying a set of translation protocols. To that end, each layer of significance regarding the city is being translated into georeferenced information fields (pic.02) based on the number of points populating a specific area and the proximity of these points. The layers' superimposition allows for their varying intensities and densities to emerge (pic. 03), and while the complexity of information remains high, the mappings' representational efficacy allows for the receiver to better perceive and connect with the available information. As new layers of significance enter the virtual map, urban constellations change and adapt informing their structures and information flows between the system and the city.

The mapping methodology proposes that viewing of an object becomes possible from within the object and not only from an exterior viewpoint. By mapping a landscape of interacting points, we have developed a mechanism that extracts information from contemporary urban landscapes without theorizing a single viewpoint as a vantage point. Any geolocated point within the urban sphere becomes then a vantage point of the same value independently of our preconceived symbolic structures. The city ceases to have its own - a priori - coordinate system (the roads or the built structures) and acquires a new one based on its interaction with the receiver. An open system in the process of formation.

This abstraction process aims at complementing the site specific content of significance by qualitatively compressing the available information. The concept of abstraction as a site specific operation is crucial because of its immanent capacity to compress different informational layers in a single entity while at the same time preserving their invariant traits and hence their capacity to diversify into local instantiations. Hecate is in that respect a method for organizing available information, simplifying its representation in a way that it can be perceived or explained, finally diversifying thinking processes and increasing possibilities for new territories of spatial interaction to emerge. After the end of this process, Hecate visualizes space as a connectionist model, by means of the representation of the 
interconnections of its parts but without any of its parts. Space is thus differentially defined as being composed of relational fields - both actual and virtual ones - as well as by their interactions. In that light, Hecate is introduced as a cognitive mechanism for the virtual and actual city.

\section{Hecate: an Infrastructural System}

Each time, an instantiation of an autonomous architectural object is extracted and then reinserted into the city thus creating a new materialized informational network. This network points to areas within the urban context that are highly significant, places where intensities and densities of information merge and overlap. Hecate's physical model is a direct result of those interactions within the global information system of the city. Continuously performing a recurring process of collecting information and re-distributing it in the city, it creates a virtual network of nodes that interact and behave as a "living" organism attached to the actual city. Hecate manifests itself as both an analog and digital suspended framework that sketches out an entirely new map of the territory, a continuous, undetermined and homogeneous network, affording the city the promise of unlimited communication and social interaction.

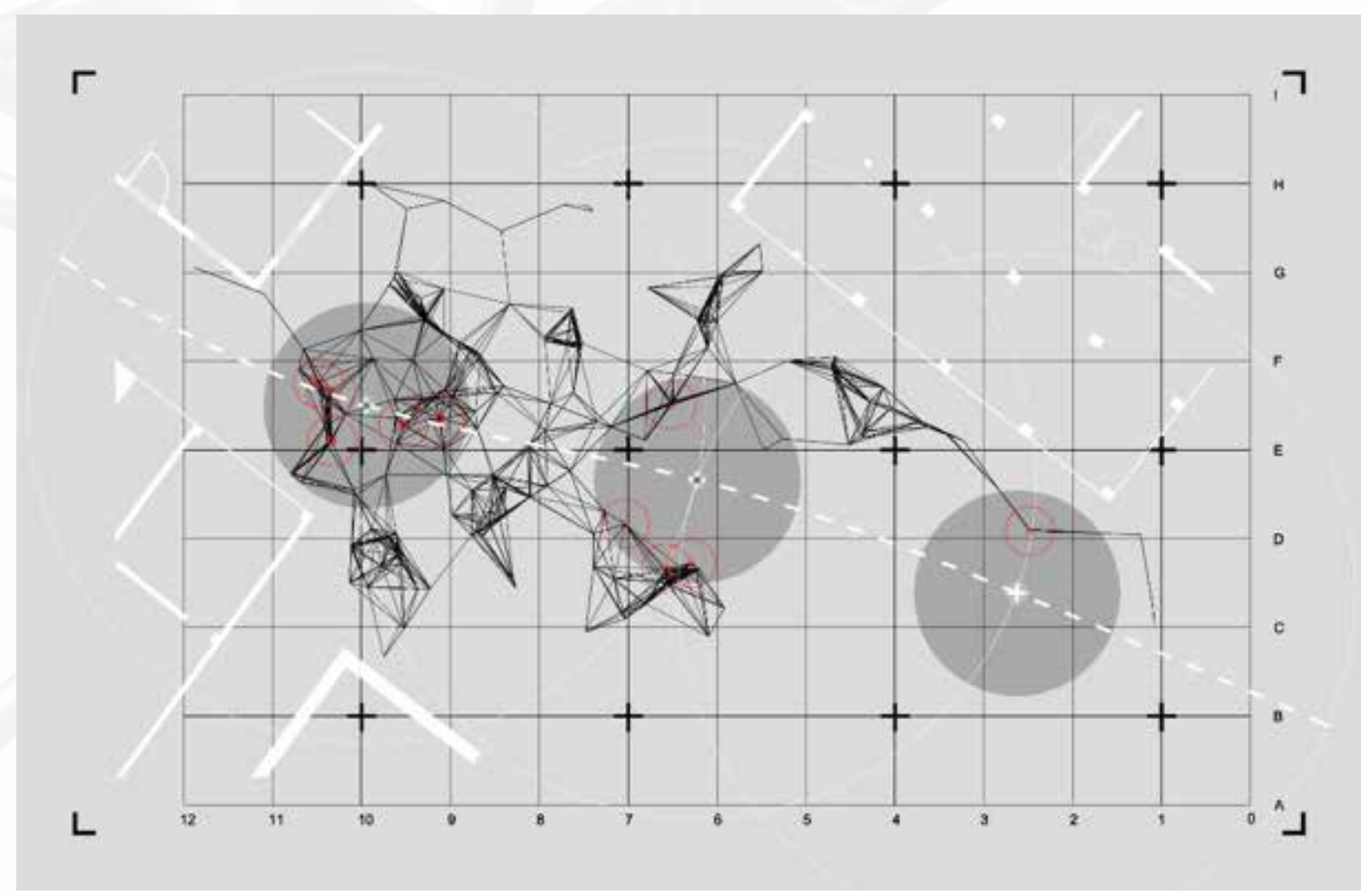

Picture 04: Physical instantiations (Passia \& Roupas, 2018).

Hecate is digitally and physically realized as an interactive connectionist city scape (pic.05), a connectionist model, with synapses of varying strength, representing the amount of information incorporated as active system passageways. This informative spatial object consists of a network of interconnected nodes, which retain their ability to change according to local information. They are also able to interconnect with other nodes, self-reproduce themselves or withdraw from the system completely, revealing their immanent capacity for life. Hecate's organizational structure has the performance capacity of an elastic urban fabric that associates, disassociates, controls, determines, consumes existing structures within the city. 

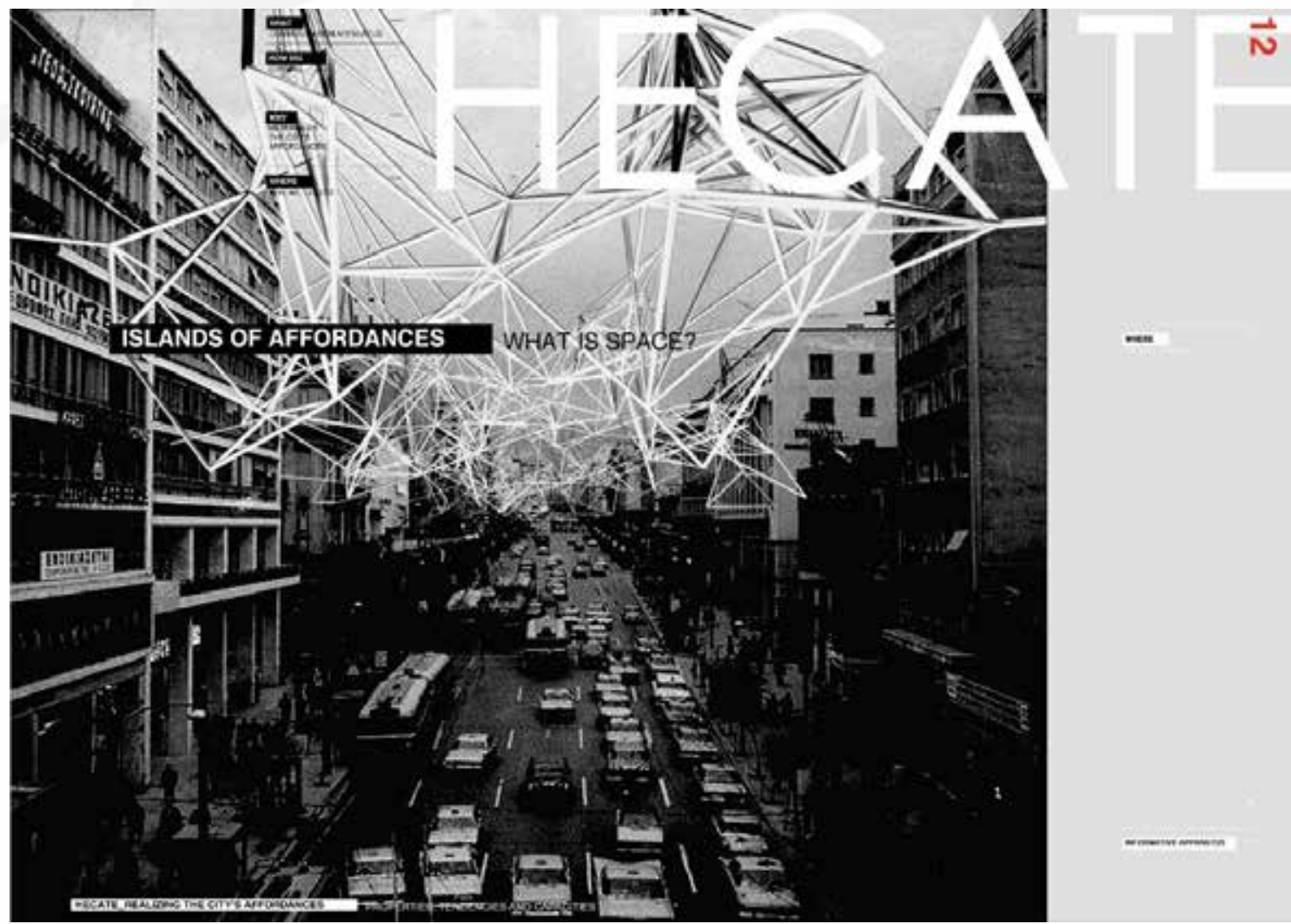

Picture 05: Hecate: Interaction protocol (Passia \& Roupas, 2018).

Hecate is composed of four parts that connect the physical and digital realms to further enhance Hecate's impact as a visual thinking tool. It is composed of a database, an array of sensors attached to the city's built structures, a large number of AR units located in the city, and a small number of physical models of Hecate's structure. In this respect, Hecate can be experienced within the cityscape both digitally and physically, through the augmented reality units and Hecate's physical models, respectively. A large number of augmented reality units are scattered in specific areas of the city that facilitate the depiction of the available in situ information and enhance Hecate's implementation into the cityscape. Hecate's material manifestation consists of a small number of physical models in human scale where one may experience the space produced through Hecate's net-like structure.

At this point, Hecate is no longer regarded as an exteriority to the city, but as a constitutive element of contemporary urban complexity. As it monitors the city's networks of significance in time, it infiltrates the cityscape to become its most intricate infrastructural system, one that merges with its extensive structures to visualize the city's reality. Within the contemporary social and spatial complexity there is no specific vantage point from where one is able to perceive the networks of information within the global milieu. Hecate intervenes at that exact point as an active, interactive networked landscape with both a physical and virtual form, as a space within which one is able to grasp the complexity of the city in terms of networked information fields. Hecate mediates a dynamic, interactive relationship between space and society: an integral part of their complexity coming into being, of a local and at the same time a global ecosystem of relations. 


\section{Bibliography}

Deleuze, G., \& Guattari, F. (1987). A Thousand Plateaus: Capitalism and Schizophrenia. Minneapolis: University of Minnesota Press.

Delanda, M. (2006). A New Philosophy of Society: Assemblage Theory and Social Complexity. London and New York: Continuum.

DeLanda, M. (2013). Intensive Science and Virtual Philosophy. London and New York: Continuum.

DeLanda, M. (2014). Possibility Spaces: Manuel DeLanda in conversation with Christoph Cox. In C. Cox, J. Joskey and S. Malik (Eds.), Realism, Materialism, Art, (pp. 87-96). Berlin: Sternberg Press.

Gibson, J. J. ([1979]1986). The Ecological Approach to Visual Perception. New Jersey: Lawrence Erlbaum Associates.

Meillassoux, Q. (2012). Iteration, Reiteration, Repetition: A Speculative Analysis of the Meaningless Sign [Lecture at Free University, Berlin, 20 April 2012]. Retrieved from https://cdn.shopify.com/s/files/1/0069/6232/files/Meillassoux_ Workshop_Berlin.pdf. Accessed on December 132018.

Meillassoux, Q. (2014). Time Without Becoming. Edited by Anna Longo. Mimesis International.

Negarestani Reza. (2014). Torture Concrete : Jean-Luc Moulene and the Protocol of Abstraction. New York: Sequence Press.

Wikipedia contributors. (2019, January 4). Hecate. In Wikipedia, the Free Encyclopedia. Retrieved from https:// en.wikipedia.org/w/index.php?title=Hecate\&oldid=876787628. Accessed on January 42019.

Cite this article as: Passia Y., Roupas P., "The Affective City: Cartography of Machinic Urban Assemblages", International Conference on the $4^{\text {th }}$ Game Set and Match (GSM4Q-2019), Doha, Qatar, 6-7 February 2019, https:// doi.org/10.29117/gsm4q.2019.0014 